\title{
Tissue Engineering - Collagen Matrix Based Flaps - a Simple Construct Experimental and Theoretical Aspects
}

\author{
SIDONIA SUSANU ${ }^{1}$, ANDREEA MORARU², DAN COSTIN², CRISTINA MARCELA RUSU³, MARICEL AGOP3,4*, GABRIELA CIOCA ${ }^{5}$ \\ ${ }^{1}$ Grigore T. Popa University of Medicine and Pharmacy, Department of Plastic and Reconstructive Surgery, 16 Universitatii Str., \\ 700115, Iasi, Romania \\ ${ }^{2}$ Grigore T. Popa University of Medicine and Pharmacy, Department of Ophthalmology, 16 Universitatii Str., 700115, Iasi, Romania \\ ${ }^{3}$ Gheorghe Asachi Techical University of lasi, Department of Physics, 73 D. Mangeron Blvd., 700050, Iasi, Romania \\ ${ }^{4}$ Romanian Scientists Academy, 54 Splaiul Independentei Blvd., 050094, Bucharest, Romania \\ ${ }^{5}$ Lucian Blaga University of Sibiu, Faculty of Medicine, Periclinical Department, Victoriei Blvd., 550024, Sibiu, Romania
}

\begin{abstract}
In our study we demonstrate the possibility to create a free flap using only a collagen matrix and a vascular pedicle. The processes of cell migration and differentiation, endogenous collagen synthesis, vascularnetwork development, all rely on the arteriovenous loop introduced in the scaffold with no participation of surrounding vascularity. The flap has the capability to revascularize a full thickness skin graft, and also to establish vascular connections with adjacent vascular territories. Also, a fractal mathematical model was built based on spontaneous breaking symmetry to explain the behaviors of biostructures mentioned above.
\end{abstract}

Keywords: tissue engineering, collagen matrix, neovascularisation, free flap

The ultimate goal of reconstructive surgery is the reestablishment of anatomical and functional integrity of post traumatic, posttumoral or malformative defects, with regard for esthetic considerations. With the introduction of the new techniques of free tissue transfer a new era of reconstructive surgery was opened. The most complex combination of tissues, including skin, vessels, nerves, muscles, cartilage and bone can now be ensembled and free transferred in the desired place.

In order to reduce the morbidity of donor site, as well as for better adapt the reconstructive solutions to the defect to be reconstructed, there has been introduced the concept of flap prefabrication $[3,4]$. The prefabricated flaps have the advantages of reducing the donor site morbidity, permitting to obtain flaps better adapted to the recipient site requirements, safer and easier surgical techniques [511].

The techniques of tissue engineering have manifested a rapid development in the last decades. A step forward in this matter represents the obtaining of neovascularised flap, based on a vascular carrier and a collagen matrix. This new generated tissue should be able to survive based only on its pedicle and also to establish vascular anastomosis with surrounded tissues. Thus the flaps could be used for coverage of defects with poor vascularity [12-18].

Our study aims to describe a simple technically construct for obtaining neovascularised flaps, based on collagen matrix, growth factors and a vascular pedicle. Our work demonstrate the possibility to transform the collagen scaffold in neovascularised, living tissue, based exclusive on saphenous pedicle, with no participation of the surrounding tissue vascularity.

\section{Experimental part}

Materials and methods

Laboratory Wistar rats weighing 350-400 g underwent operation. All the experiments were conducted according to National and Medical Research Guidelines for Animal Welfare. The inferior abdomen and the posterior limb were shaved and preoperatory drawn marked the femoral and

saphenous arteries and veins, groin ligament, the incision line and the further place of the flap (Fig. 1).

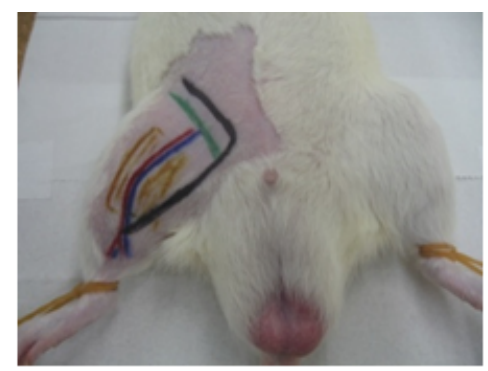

Fig. 1. Preoperatory drawing

Skin incision has a reverse $L$ shape and was carried down to fascia, the cutaneous flap was retracted laterally with exposure of saphenofemoral pedicle. The saphenous vessels were dissected from distal end to their emergence from femoral vessels. The femoral vessels were dissected proximal until the groin ligament. The distal end of saphenous artery and medial saphenous vein were ligated and the vessels were cut $3 \mathrm{~mm}$ proximal. An end-to-end anastomosis was performed with 11/0 sutures, using a standard antero-posterior technique.

Pieces of collagen matrix of $3 / 2 \mathrm{~cm}$ were socked with autologous growth factors. The vascular loop was included in the depth of the scaffold and the collagen tissue was sutured (Fig. 2). The template was fixed with its silicon layer posterior while the collagen layer anterior thus permitting a better evaluation of the flap evolution.

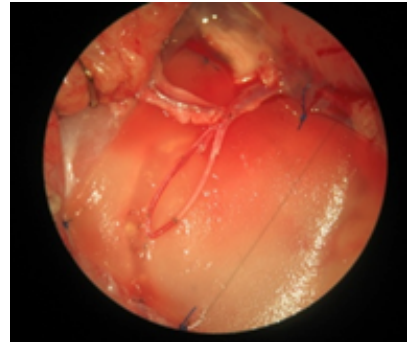

Fig. 2. The experimental model

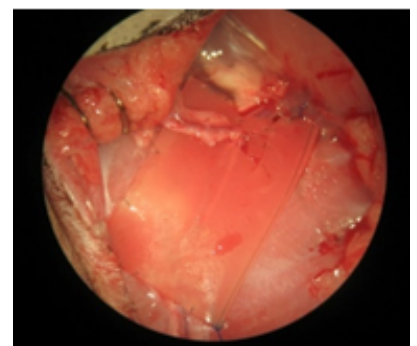

Fig 3. The construct fixed to the groin ligament 
The flap was wrapped with polyethylene sheet thus isolating both surfaces and all four edges from surrounding tissues. A single hole of $3 \mathrm{~mm}$ on the proximal edge permits the pedicle to enter into the scaffold. The construct was fixed with $5 / 0$ sutures to the groin ligament and to the anterointern muscles of the thigh (Fig. 3).

The skin flap was placed in its position, over the construct, and sutured with 5/0 interrupted sutures.

The flap was evaluated at 7, 14, 21, 28 days, 3 months after implantation.

At 28 days of evolution the new vascular net formed within the flap was demonstrated by bleeding inducing test at the edge of the flap and by injecting of India ink into the pedicle, proximal to the flap.

The pedicle was isolated from the surrounding tissue. The construct (the polyethylene sheets including the flap) was raised from its place and isolated from surrounding tissue. The anterior polyethylene sheet was removed, for a better evaluation of the flap surface. A $2 \mathrm{~mm}$ incision performed at the distal edge of the flap induce bleeding at this level, thus demonstrating the perfusion based exclusively on the pedicle.

The femoral artery was catheterized with a 28 gauge butterfly needle $10 \mathrm{~mm}$ proximal to the emergence of saphenous artery. India ink was injected into arterial trunk. The ink perfused the branches arising from the pedicle and subsequently the tissue itself, thus demonstrating the direct dependence between the distal, new formed tissue and the pedicle.

At 28 days of evolution we could demonstrate the capacity of the new vascular netw ork to establish vascular anastomosis with the surrounding tissue and also to permit perfusion of poor vascular tissue around the flap. On this purpose we apply a full thickness skin graft on the anterior surface of the flap, while the posterior surface remained isolated from the underlying tissue. The flap was perfused only from its saphenous pedicle. The skin overlying the flap was harvested as a full thickness skin graft. The skin was applied on the anterior surface of the flap and sutured to the flap and to the surrounding skin.

After 14 days the skin graft was fully taken with pink color and a normal consistence.

The histology examination performed at 7, 14, 21, 28 days indicated also the graduated evolution of the collagen matrix toward neovascularised living tissue. The exam at 3 months confirmed the stability of new formed tissue.

\section{Results and discussions}

We observed the generation of a new, neovascularised tissue, concomitant with progressive resorption of the collagen matrix.

At 7 days after implantation from the saphenous pedicle new formed branches could be observed. The branches arise from both artery and vein, with small, if none difference on the number between arterial or venous site. At this time the neovascularisation process is concentrated around the pedicle

At 14 days the neovascularisation process extends to more than half of the flap surface. The pedicle is still obvious in the depth of the scaffold. The color of the collagen matrix transform toward orange.

At 21 days of evolution almost the entire surface of the flap had a red color, with the vascular net obvious on its surface.

At 28 days the entire surface of the flap had a homogenous, red color. The vessels are no more obvious on its surface with direct observation. The examination under digital exploratory microscope with polarization the vascular netw ork became evident.
The perfusion of the flaps could be demonstrated based only on the saphenous pedicle using the induced bleeding test at the edges of the flap. Also, injecting of India ink into the femoral artery, $10 \mathrm{~mm}$ proximal to the emergence of the saphenous artery produced the perfusion of the ink first into the main branches and subsequently to the small vessels, at the distal edges of the flap.

From each group 2 flaps were skin grafted with full thickness skin graft, harvested from the internal surface of the tight. The posterior surface of the flaps was keptisolated from the underlying tissues. After 14 days the pink aspect of the grafts indicated the complete taken of the grafts.

The grafted flaps were raised on their pedicle, together with an excess of about $5 \mathrm{~mm}$ form the surrounding skin. Injecting of India ink in the femoral artery determined the black coloration of the exceeded skin, thus demonstrating the vascular anastomosis between the vessel of the graft and the surrounding skin (Fig. 4).

\section{Mathematical model}

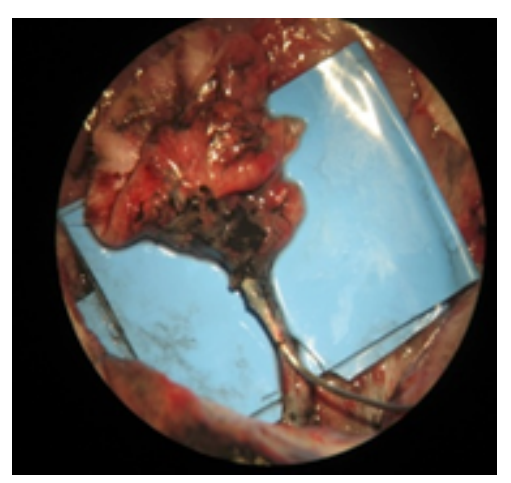

Fig 4. The perfusion test

Since the only possible physical mechanism by which all complex systems can create mass is the spontaneous breaking symmetry, in the following we will admit the functionality of such a mechanism by calibrating it on our experimental data. For this purpose let us suppose the following:

i) the sponge collagen -vascular loop assembly must be assimilated to a fractal medium (informational matrix). Indeed, on the informational matrix, blood vessels grow slowly and chaotic, which gives it both structural and functional characteristics of fractal type [19];

ii) the dynamics of the fractal medium are described by the fractal state function $\Psi[20,21]$ with complex scalar field functionality. This function is dependent on both 4 coordinates $X^{\mu}$ and the scale resolution $d \tau$,

$$
\Psi=\psi\left(X^{\mu}, d \tau\right)
$$

iii) the presence of the growth factor must be assimilated to an external constraint on the fractal medium, in which case the mechanism of spontaneous breaking symmetry becomes functional. Then the process of blood vessel growth becomes non-chaotic, accelerated and leaded by the fractal medium;

iv) on the fractal medium, in the absence of the growth factor, the information:

$$
I=\ln \Psi^{\prime} \bar{\psi}
$$

with $\Psi$ the complex conjugated of $\Psi$, is implicit and unmanifest, while in the presence of the growth factor it becomes explicit and manifest. For other details, see [2224];

v) we prove that on a fractal medium, the implicit-explicit transition of information implies the fractal type spontaneous breaking symmetry (for general details, see [25-27]). Then, a specific calibration becomes functional, 
calibration which enables a holographic implementation of the interaction entity-fractal medium (for example celltissue). Through this mechanism, the no-mass particles of biological type (the fractal goldstones of biological type) are eliminated and the particles with mass of biological type are generated.

\section{Fractal type spontaneous breaking symmetry}

Fractal type gauge theories have the property that all the components of the fractal type gauge fields have null fractal type mass. In fact, there exist both fractal type gauge fields having null fractal type mass, and fractal type gauge fields having nonzero fractal type mass. The mere additon of a fractal type mass term in the expression of the fractal type gauge Lagrangean does not solve the problem since the fractal type gauge symmetry is violated through this. For solving the mass problem of the fractal type gauge fields it is necessary the development of a fractal type model with fractal type spontaneous breaking symmetry, in which the fractal type Lagrangean remains fractal type gauge invariant, but the fractal medium state is no more fractal type invariant. This is the fractal model of the fractal type spontaneous breaking symmetry. In this context, we will prove that one part of the fractal type gauge fields gains fractal type mass, while another part has no fractal type mass.

The degeneration of the fractal medium state and fractal type breaking symmetry

Let us consider a fractal type system described by a fractal type Lagrangean $L$ or, equivalently, by a fractal type Hamiltonian $\mathrm{H}$. The fractal type eigenstates of $\mathrm{H}$ can be described by means of the geodesics and, based on the standard procedure [21], by the fractal type equation:

$$
H \Psi_{n}=E_{n} \Psi
$$

Every fractal type state of the fractal type system is specified through a value $E_{0}$ of the fractal type energy and through the fractal type stafe function $\Psi_{v}$.

The state of minimal fractal type energy $E_{0}$ described by the fractal type state function $\Psi_{0}$ is called a fractal medium state. If to $\mathrm{E}_{0}$ corresponds a unique fractal type state 0 , then the fractal medium state is called a nondegenerated fractal type state; otherwise, the fractal medium state is called a degenerated fractal type state.

The fractal medium state $\Psi$ is called fractal type invariant to a fractal type group $G$ of transformations, if this state turns itself into itself with respect to this group; otherwise, it is said to be fractal type non-invariant. However, there exists a close relation between the fractal medium state invariance with respect to a group of transformations $\mathrm{G}$ and the invariance of the Lagrangean with respect to the same group (Coleman's theorem [ 2527]), in the framework of relativistic quantum field theories. We shall extend this result for the fractal type fields theory in the following form.

If the fractal medium state is fractal type invariant, then also the fractal type Lagrangean should be necessarily fractal type invariant (the fractal medium state invariance specifies the fractal type invariance of the fractal type Universe). In this case, the fractal type system has an exact symmetry of fractal type. If the fractal medium state is not fractal type invariant, then the fractal type Lagrangean can be fractal type invariant or fractal type non-invariant. In the first case, one asserts that the fractal type symmetry is fractal type explicitely broken, while in the second case, the fractal type symmetry is said to be fractal type spontaneously broken. We shall prove that the fractal type spontaneous breaking of the fractal type symmetry necessarily implies the generation of fractal type particles having null fractal type mass. Practically speaking, one extends Goldstone's theorem to fractal type spatialtemporal manifolds, the fractal type particles having null fractal type mass being called fractal type goldstons. In what follows, we shall make explicit such situations. The spontaneous breaking of the fractal type symmetries will be completed by the fractal type Higgs mechanism, which will enable us to eliminate the fractal type golstons and to associate fractal type mass to fractal type gauge fields.

The spontaneous breaking of the fractal type global symmetries

Let us consider first the fractal type global symmetry described by the fractal type abelian group U(1). Let be then the Lagrangean:

$$
L=\left(\partial_{\mu} \bar{\Psi}\right)\left(\partial^{\mu} \Psi\right)-m^{2} \bar{\Psi} \Psi-\frac{f}{4}(\bar{\Psi} \Psi)^{2}, \mu=1,2,3,4
$$

where $\Psi(X)$ is a fractal type complex scalar field, $\Psi(X)$ is the complex conjugate of $\Psi(X), X^{0}, X^{\mu}$, are the fractal 4coordinates of a point of a fractal space-time manifold, $\partial_{\mu}$ is the scale covariant derivative $[20,21], f>0$ is the fractal type coupling constant of this field and $m$ is the fractal type mass associated to the field.

Let us note that the Lagrangean (2) can be written with respect to the implicit information in the form:

$$
L=\left(\partial_{\mu} \bar{\Psi}\right)\left(\partial^{\mu} \Psi\right)-m^{2} \exp (I)-\frac{f}{4} \exp ^{2}(I)
$$

Then, the potential:

$$
V(I)=m^{2} \exp (I)+\frac{f}{4} \exp ^{2}(I)
$$

is a function depending only on the implicit information.

The Lagrangean (2) is invariant with respect to the fractal type global group U(1), whose fractal type transformations (fractal type global transformations U(1)) are given by the relations:

$$
\begin{aligned}
& \Psi(X) \rightarrow \Psi^{\prime}(X)=e^{-i g \varepsilon} \Psi(X) \\
& \bar{\Psi}(X) \rightarrow \bar{\Psi}^{\prime}=e^{-i g \varepsilon} \bar{\Psi}(X)
\end{aligned}
$$

Let us write now the Hamiltonian $H$ associated to the Lagrangean $L$. For this purpose, we introduce the fractal type momentum associated to $\Psi$ and $\Psi$ through the definition relationships:

$$
\pi(X)=\frac{\partial L}{\partial \dot{\psi}^{\prime}}, \bar{\pi}(X)=\frac{\partial L}{\partial \dot{\Psi}^{\prime}} \dot{\Psi}=\frac{\partial \psi}{\partial t}, \dot{\bar{\Psi}}=\frac{\partial \bar{\Psi}}{\partial t}
$$

The Hamiltonian of these fields has then the form:

$$
H=\pi \dot{\Psi}+\bar{\pi} \dot{\bar{\Psi}}-L \equiv E
$$

Substitutina (2) in (5), with the notations , $\dot{\Psi}=\partial_{0} \Psi, \dot{\bar{\Psi}}=\partial_{0} \bar{\Psi}$, we obtain the expression:

$$
\begin{gathered}
E=\frac{\partial L}{\partial\left(\partial_{0} \Psi\right)} \partial_{0} \Psi+\frac{\partial L}{\partial\left(\partial_{0} \bar{\Psi}\right)} \partial_{0} \bar{\Psi}- \\
-\left[\left(\partial_{0} \bar{\Psi}\right)\left(\partial_{0} \Psi\right)-\left(\partial_{l} \bar{\Psi}\right)\left(\partial_{l} \Psi\right)-m^{2} \bar{\Psi} \Psi-\frac{f}{4}(\bar{\Psi} \Psi)\right]
\end{gathered}
$$

or even more,

$$
\begin{aligned}
& E=\left(\partial_{0} \bar{\Psi}\right)\left(\partial_{0} \Psi\right)+\left(\partial_{l} \bar{\Psi}\right)\left(\partial_{l} \Psi\right)+m^{2} \bar{\Psi} \Psi+\frac{f}{4}(\bar{\Psi} \Psi)^{2}= \\
& =\left(\partial_{0} \bar{\Psi}\right)\left(\partial_{0} \Psi\right)+\left(\partial_{l} \bar{\Psi}\right)\left(\partial_{l} \Psi\right)+m^{2} \exp (I)-\frac{f}{4} \exp ^{2}(I)
\end{aligned}
$$

The minimum value of the fractal type energy $E$ of $\Psi(X)$, i.e., the value corresponding to the fractal medium state results by the conditions: 
$\frac{\partial E}{\partial \Psi}=m^{2} \bar{\Psi}+\frac{f}{2} \bar{\Psi}^{2} \Psi=0, \frac{\partial E}{\partial \bar{\psi}}=m^{2} \Psi^{\prime}+\frac{f}{2} \bar{\psi} \Psi^{2}=0$

These conditions yield to the fractal medium state:

$$
\Psi_{f}(X)=0, \bar{\Psi}_{f}(X)=0
$$

If we define the fractal type potential associated to $\Psi(X)$ through the relation:

$$
V(I)=m^{2} \bar{\Psi} \Psi+\frac{f}{4}(\bar{\Psi} \Psi)^{2}=m^{2} \exp (I)+\frac{f}{4} \exp ^{2}(I)
$$

then we observe that the fractal medium state thus obtained is unique (non-degenerated) and stable. Additionally, it is invariant with respect to the fractal type global transformations given by the relations (3). The Lagrangean $L$ is also invariant with respect to these transformations.

In conclusion, the model described by the Lagrangean (2) has a fractal type global exact symmetry U(1), with the fractal medium state $\Psi_{f}(X)=0, \bar{\Psi}_{f}(X)=0$, nondegenerated and stable.

The spontaneous breaking of the fractal type global symmetry group

Let us consider now the Lagrangean:

$$
\begin{aligned}
& L_{1}=\left(\partial_{\mu} \bar{\Psi}\right)\left(\partial^{\mu} \Psi\right)+m^{2} \bar{\Psi} \Psi-\frac{f}{4}(\bar{\Psi} \Psi)^{2}= \\
& =\left(\partial_{\mu} \bar{\Psi}\right)\left(\partial^{\mu} \Psi\right)+m^{2} \exp (I)-\frac{f}{4} \exp ^{2}(I)
\end{aligned}
$$

which differs from (2) by the changed sign in the second term from the right side. This Lagrangean is also invariant with respect to the fractal type global transformations $U(1)$ and this fact can be easily checked.

As it was stated before, the fractal medium state is obtained by the conditions:

$$
\begin{aligned}
\frac{\partial E}{\partial \Psi} & =-m^{2} \bar{\Psi}+\frac{f}{2} \bar{\Psi}^{2} \Psi=0, \frac{\partial E}{\partial \bar{\Psi}}= \\
& =-m^{2} \Psi+\frac{f}{2} \bar{\Psi} \Psi^{2}=0
\end{aligned}
$$

Here, the fractal medium state corresponds to the value:

$$
\bar{\psi} \Psi=\frac{2 m^{2}}{f},\left|\Psi^{\prime}\right|=\frac{\sqrt{2} m}{\sqrt{f}} \equiv R,
$$

and the values

$$
\Psi^{\prime}=0, \bar{\Psi}=0
$$

correspond to a fractal type maximum.

The fractal system described by the Lagrangean $L_{1}$ has now an infinity of fractal medium states, namely, all points from the fractal type circle of radius $R$ from the fractal type complex plane $(\operatorname{Re} \Psi ; \operatorname{Im} \Psi)$. Thus, in this case, the fractal medium state is infinite degenerated.

The transformations ( 3 ) lead a fractal medium state (a point from the circle of radius $R$ ) to another fractal medium state (another point from the same circle) and thus the fractal medium state is no more invariant with respect to the fractal type global symmetry group $U(1)$. Although, the Lagrangean remains invariant with respect to this group and so we have a spontaneously broken fractal type global symmetry U(1).

One can easily check that all the fractal medium states obtained in this way are stable, i.e., small variations of $\Psi(X)$ around a fractal medium state do not remove the fractal type system from the state of equilibrium (it returns to the state of equilibrium).

In order to build a self-consistent model, we mustchoose a unique, stable fractal medium state, i.e., to choose a certain, well-defined point, from the circle of radius $R$. We shall choose this point and we shall move the origin of the fractal type system in this point. Thus we shall obtain the coordinate system $\left(\Psi_{1}, \Psi_{2}^{\prime}\right)$.

In the initial coordinate system, $\Psi(X)$ writes in the form:

$$
\Psi(X)=\frac{1}{\sqrt{2}}\left(\frac{2 m}{\sqrt{f}}+\Psi_{1}(X)+i \Psi_{2}(X)\right)
$$

where the factor $1 / \sqrt{2}$ has been chosen conveniently for our purposes and $\psi_{1} X$ and $\psi_{2} X$ are two fractal type real scalar fields. Substituting (12) in (10) we get:

$$
\begin{aligned}
L_{1}(\Psi) \rightarrow L_{2}\left(\Psi_{1}, \Psi_{2}\right)= & =\frac{1}{2}\left(\partial_{\mu} \Psi_{1}\right)^{2}+\frac{1}{2}\left(\partial_{\mu} \Psi_{2}\right)^{2}+\frac{m^{2}}{2}\left(\frac{4 m^{2}}{f}+\frac{4 m}{\sqrt{f}} \Psi_{1}+\Psi_{1}^{2}+\Psi_{2}^{2}\right) \\
& -\frac{f}{16}\left(\frac{16 m^{4}}{f^{2}}+\frac{16 m^{2}}{f^{2}} \Psi_{1}^{2}+\Psi_{1}^{4}+\Psi_{2}^{4}+\frac{32 m^{3}}{f \sqrt{f}}+\frac{8 m^{2}}{f} \Psi_{1}^{2}+\frac{8 m^{2}}{f} \Psi_{2}^{2}\right. \\
& \left.+\frac{8 m^{2}}{\sqrt{f}} \Psi_{1}^{3}\right)-\frac{f}{16}\left(\frac{8 m}{\sqrt{f}} \Psi_{1} \Psi_{2}^{2}+2 \Psi_{1}^{2} \Psi_{2}^{2}\right) \\
& =\frac{1}{2}\left(\partial_{\mu} \Psi_{1}\right)^{2}+\frac{1}{2}\left(\partial_{\mu} \Psi_{2}\right)^{2}-m^{2} \Psi_{1}^{2}-\frac{f}{16}\left(\Psi_{1}^{4}+\Psi_{2}^{4}+2 \Psi_{1}^{2} \Psi_{2}^{2}\right) \\
& -\frac{m \sqrt{f}}{2}\left(\Psi_{1}^{2}+\Psi_{2}^{2}\right) \Psi_{1}+\frac{m^{4}}{f}
\end{aligned}
$$

or, renouncing to the constant term $\frac{m^{4}}{f}$, which does not modify the dynamics described by the considered Lagrangean, we get the following equivalent expression:

$$
L_{2}\left(\Psi_{1}, \Psi_{2}\right)=\frac{1}{2}\left(\partial_{\mu} \Psi_{1}\right)^{2}-\frac{m_{1}^{2}}{2} \Psi_{1}^{2}+\frac{1}{2}\left(\partial_{\mu} \Psi_{2}\right)^{2}-\frac{f}{16}\left(\Psi_{1}^{4}+\Psi_{2}^{4}+2 \Psi_{1}^{2} \Psi_{1}^{2}\right)-\frac{m \sqrt{f}}{2}\left(\Psi_{1}^{2}+\Psi_{2}^{2}\right) \Psi_{1}
$$


where $m_{1}=\sqrt{2} m$ is the fractal type mass of the fractal type scalar particle associated to the $\Psi_{1}(X)$ field.

The Lagrangean $L_{2}\left(\Psi_{1}, \Psi_{2}\right)$ does not contain a fractal type mass term (which is directly proportional to $\Psi_{2}^{2}(X)$, i.e., the fractal type scalar particle described by $\Psi_{2}(X)$ has null fractal type mass (we shall call it a fractal type goldstone). This is a result of the fractal type spontaneous breaking symmetry, for the case of the Lagrangean $L_{2}\left(\Psi_{1} ; \Psi_{2}\right)$.

In this way, the model described by the Lagrangean $L_{1}(\Psi)$ has a fractal type spontaneously broken global symmetry $U(1)$. Consequently, $\Psi(X)$ has been converted in a fractal type real scalar field $\Psi_{1}(X)$ with the fractal type mass $m=\sqrt{2} m$ and a fractal type goldstone $\psi_{2} \quad X$ with null fractal type mass. We highlight the fact that the Lagrangean $L_{1}$ and $L_{2}$ are completely equivalent, both describing the same dynamics of the considered fractal type system.

Comparing the two examples with $L_{1}$ and $L_{2}$, one observes that there exists a fractal type critical value $\left(\mathrm{m}^{2}=0\right)$ which determines if the fractal type spontaneous breaking symmetry takes place or not. Thus, if in the Lagrangean we have the term with- $m^{2}$, there exists a stable and non-degenerated fractal medium state of the fractal system, $\Psi_{f}(X)=0$, and the fractal type global symmetry is exact. If in the Lagrangean we have the term with $+m^{2}$, the fractal medium state is infintely degenerated and noninvariant, $\Psi_{f}(X)$, the fractal type global symmetry being fractal type spontaneously broken.

It seems that the simple change of sign for the term with $\mathrm{m}^{2}$ from the Lagrangean (2) means the insertion into the model of certain non-physical fractal type particles with fractal type imaginary mass Hnwever, itshould be clarified the fact that the term $m^{2} \psi^{\prime} \Psi$ from the Lagrangean corresponds to a fractal type mass term only if the fractal state $\Psi=0$ is a fractal type stable equilibrium position. For the case of the Lagrangean $L_{1}(\Psi)$, the fractal type potential

$$
V(\Psi)=-m^{2} \bar{\psi} \Psi+\frac{f}{4}(\bar{\Psi} \Psi)^{2}=-m^{2} \exp (I)+\frac{f}{4} \exp ^{2}(I)
$$

has a fractal type maximum in $\Psi=0$ and thus the term $m^{2} \bar{\psi} \Psi$ is not a fractal type mass term. In $\Psi=0$ the fractal type system is not stable, while in $|\Psi|=\frac{\sqrt{2} m}{\sqrt{f}}$ it is stable.

In order to determine the fractal type physical masses of the fractal type particles, we must use the series expansion of the fractal type potential $\mathrm{V}(\Psi)$ in the neighborhood of the fractal type minimum:

$$
\Phi_{1}^{\prime} \equiv \eta+v, v=\frac{\sqrt{2} m}{\sqrt{f}} \equiv R, \Phi_{2}^{\prime} \equiv \xi=\Phi_{2}+0
$$

where $\eta$ and $\xi$ are fractal type infinitesimal quantities. Then, the Lagrangean $L_{2}$ becomes:

$$
L_{2}=\frac{1}{2}\left(\partial_{\mu} \eta\right)^{2}+\frac{1}{2}\left(\partial_{\mu} \xi\right)^{2}-m^{2} \eta^{2}+0 \cdot \xi^{2}+\cdots
$$

In this expression it appears a fractal type mass term for $\eta$, but not for $\xi$. The fractal type mass of $\Psi_{1}$ (or $\eta$ ), which has the expression $\sqrt{2} m$, is real and thus the fractal term

$\frac{1}{2} m_{1}^{2} \Psi_{1}^{2}$ from the Lagrangean $L_{2}$ has the correct sign

(minus).
In consequence, in the case of the Lagrangean $L_{2}, \Psi_{11}$ has a physical sense and not $\Psi(X)$.

We describe a simple technically construct for obtaining neovascularised flaps, based on collagen matrix, growth factors and a vascular pedicle. In such context, we demonstrate the possibility to transform the collagen scaffold in neovascularised, living tissue, based exclusive on saphenous pedicle, with no participation of the surrounding tissue vascularity. Such experimental behaviors of the biostructures mentioned above can be correlated with a fractal mathematical model based on spontaneous breaking symmetry. For the moment, this can describe just qualitatively the biostructures dynamics, without making them explicit, both in terms of processes and mechanisms that generate them, as well as of their nature. However, a quantitative description implies the calibration of the model parameters (in our case the parametersm and $f$ from relation (2)) to the type of biostructure, thus specifying both the resolution of the scale and the corresponding gauge. But, specifying the gauge involves in vivo determinations which, in general, are very difficult.

\section{Conclusions}

We described a method to obtain a vascularized flap using a skin substitute as collagen matrix and a vascular loop represented by the anastomosis between saphenous artery and medial saphenous vein. The generating of the new flap was possible based only on its pedicle, with no influence of adjacent tissues vascularity.

Also, the forming of a new tissue was possible independent to the type of the collagen scaffold used (either IntegraT ${ }^{\mathrm{TM}}$, Terudermis or Pelnac).

Further studies are necessary to determine the maximal size of a flap possible to be obtained based on a specific size of the pedicle, and also to clarify the influence of growing factors.

We consider our method as a promising solution for most difficult defects, with a large applicability in clinical practice support and/or endorsement from any medical company from Romania, Japan or abroad.

A fractal mathematical model was built based on spontaneous symmetry breaking to explain the behaviors of biostructures mentioned above. In the future, the same model can be used to explain other behaviors of the biostructures such as those analyzed in [28-47].

\section{References}

1.BIRBRAIR, A., ZHANG, T., FILES, D. C., MANNAVA, S., SMITH, T., WANG, Z. M., MESSI, M. L., MINTZ, A., DELBONO, O., Stem Cell. Res. Ther., 5, no. 6, 2014, p. 122

2.DEL ANGEL-MOSQUEDA, C., GUTIERREZ-PUENTE, Y., LOPEZLOZANO, A.P., ROMERO-ZAVALETA, R.E., MENDIOLA-JIMENEZ, A., MEDINA DE LA GARZA, C. E., MARQUEZ-M M, DE LA GARZA-RAMOS, M,A, Head Face Med., 11, 2015, p. 29

3.YAO, S. T., Plast. Reconstr. Surg., 68, no. 3, 1981, p. 404

4.HOULE, J. M., NEUMEISTER, M. W., Plast. Reconstr. Surg., 120, no. 5, 2007, p. 1322

5.MOIEMEN, N. S., VLACHOU, E., STAIANO, J. J., THAWY, Y., Plast. Reconstr. Surg., 117, no. 7, 2006, p. 1605

6.ABAI, B., THAYER, D., GLAT, P. M., Plast. Reconstr. Surg., 114, no. 1, 2004, p. 162

7.TANAKA, Y., SUNG, K., FUMIMOTO, M., TSUTSUMI, A., KONDO, S., HINOHARA, Y., MORRISON, W. A., Plast. Reconstr. Surg., 117, no. 6, 2006, p. 1860 
8.MESA, J. M., ZAPOROJAN, V., WEINAND, C., JOHNSON, T. S., BONASSAR, L., RANDOLPH, M. A., YAREMCHUK, M. J., BUTLER, P. E., Plast. Reconstr. Surg., 118, no. 1, 2006, p. 41

9.BORSCHEL, G. H., DOW, D. E., DENNIS, R. G., BROWN, D. L., Plast. Reconstr. Surg., 117, no. 7, 2006, p. 2235

10.KAWAMURA, K., YAJ IMA, H., OHGUSHI, H., TOMITA, Y., KOBATA, Y., SHIGEMATSU, K., TAKAKURA, Y., Plast. Reconstr. Surg., 117, no. 5, 2006, p. 1471

11.NEUMEISTER, M.W., WU, T., CHAMBERS C., Plast. Reconstr. Surg., 117, no. 1, 2006, p. 116

12.YAO, S. T., Plast. Reconstr. Surg., 68, no. 3, 1981, p. 404

13.KNIGHT, M. A., EVANS, G. R. D., Plast. Reconstr. Surg., 114, no. 2, 2004, p. $26 \mathrm{E}$

14.LEE, J. W., JANG, Y. C., OH, S. J., Ann. Plast. Surg., 55, no. 5, 2005, p. 500

15.BASTIDAS, N., ASHJ IAN, P. J., SHARMA, S., Ann. Plast. Surg., 62, no. 4, 2009, p. 410

16.ICHIOKA, S., OHURA, N., SEKIYA, N., SHIBATA, M., NAKATSUKA, T., Ann. Plast. Surg., 51, no. 4, 2003, p. 383

17.MOLNAR, J. A., DEFRONZO, A. J., HADAEGH, A., MORYKWAS, M. J., SHEN, P., ARGENTA, L. C., Plast. Reconstr. Surg., 113, no. 5, 2004, p. 1339

18.KOMOROWSKA-TIMEK, E., GABRIEL, A., BENNETT, D. C., MILES, D., GARBEROGLIO, C., CHENG, C., GUPTA, S., Plast. Reconstr. Surg., 115 , no. 4, 2005, p. 1010

19.MANDELBROT, B. B., The Fractal Geometry of Nature, W.H. Freeman, New York, 1983

20.NOTTALE, L., Scale Relativity and Fractal Space-Time. A New Approach to Unifying Relativity and Quantum Mechanics, Imperial College Press, London, 2011

21.MERCHES, I., AGOP, M., Differentiability and Fractality in dynamics of Physical Systems, World Scientific, Singapore, 2016

22.GRIGOROVICI, A., BACAITA, E. S., PAUN, V. P., GRECEA, C., BUTUC, I., AGOP, M., POPA, O., Entropy, 19, no. 3, 2017, p. 128

23.PREDA, C., BRANISTEANU, D., ARMASU, I., DANILA, R., VELICESCU, C., CIOBANU, D., COVIC, A., GRIGOROVICl, A., BMC Surgery, 19, 2019, p. 94

24.COBZEANU, B. M., IRIMICIUC, S., VAIDEANU, D., GRIGOROVICI, A., POPA, O., Mat. Plast., 54, no. 3, 2017, p. 531

25.CHAICHIAN, M., NELIPA, N. F., Introduction to Gauge Field Theories, Springer Verlag, Berlin Heidelberg, 1984

26.ZET, G., Simetrii Unitare si Teorii Gauge, Ed. Gh. Asachi, lasi,1998 27.DARIESCU, C., DARIESCU, M. A., Capitole de baza in Mecanica Cuantica. Microparticule si campuri, Ed. Venus, Iasi, 2007
28.IOV, T., TIMOFTE, D., DAMIAN, S. I., KNIELING, A., SCRIPCARU, C., BULGARU-ILIESCU, D., Rom. J. Leg. Med., 26, no. 2, 2018, p. 141 29.CRAUCIUC, D. V., CRAUCIUC, E. G., IOV, C. J., FURNICA, C., IOV, T., Rev. Chim.(Bucharest), 69, no. 11, 2018, p. 4088

30.HUNEA, I., DAMIAN, S. I., RADU, C. C., MOLDOVEANU, S., IOV, T., Rev. Chim.(Bucharest), 69, no. 9, 2018, p. 2482

31.PERJU, D. D., RADU, C. C., DAVID, S., IOV, T., IOV, C. J., SANDU, I., ILIESCU, D. B., Rev. Chim.(Bucharest), 69, no. 9, 2018, p. 2407

32.BACAITA, E. S., AGOP, M., Phys. Chem. Chem. Phys., 18, 2016, p. 21809

33.RADU, V., BACAITA, S.,ULINIUC, A., POPA, M., SUSANU, S., Mat. Plast., 50, no. 1, 2013, p. 18

34.MAGOP, D., BACAITA, S., PEPTU, C., POPA, M., AGOP, M., Mat. Plast., 49, no. 2, 2012, p. 101

35.GURLUI, S., AGOP, M., STRAT, M., STRAT, G., BACAITA, S., Jpn. J. Appl. Phys., 44, no. 5A, 2005, p. 3253

36.AGOP, M., ALEXANDROAIE, D., CEREPANIUC, A., BACAITA, S., Chaos Solitons Fractals, 30, no. 2. 2006, p. 470

37.AGOP, M., NICA, P., IOANNOU, P. D. ET AL., Chaos Solitons Fractals, 34, no. 5, 2007, p. 1704

38.NEJ NERU, C., NICUTA, A., CONSTANTIN, B. ET AL., J. Appl. Math., 137056, 2013

39.NEDEFF, V., MOSNEGUTU, E., PANAINTE, M. ET AL., Powder Technol., 221, 2012, p. 312

40.AGOP, M., NICA, P., GIRTU, M., Gen. Relativ. Gravit., 40, no. 1, 2008,p. 35

41.AGOP, M., MURGULET, C., Chaos Solitons Fractals,32, no. 3, 2007, p. 1231

42.GOTTLIEB, I., AGOP, M., JARCAU, M., Chaos Solitons Fractals, 19, no. 4, 2004, p. 705

43.AGOP, M., IOANNOU, P., NICA, P. ET AL., Mater. Trans., 45, no. 3, 2004, p. 972

44.AGOP, M., GRIGA, V., CIOBANU, B. ET AL., Chaos Solitons Fractals, 9, no. 7, 1998, p. 1143

45.CIUBOTARIU, C., AGOP, M., Gen. Relativ. Gravit., 28, no. 4, 1996, p. 405

46.NICULESCU, O., DIMITRIU, D. G., PAUN, V. P. ET AL., Phys. Plasmas, 17, no. 4, 2010, p. 042305

47.AGOP, M., NICA, P. E., GURLUI, S. ET AL., Eur. Phys. J. D, 56, no. 3, 2010, p. 405

$\overline{\text { Manuscript received: } 14.08 .2019}$ 